\title{
Application of Consolidation Logarithmic Fitting Method on Settlement Deformation Evaluation in Early Stage of Engineering
}

\author{
Beibei Chen ${ }^{1, \mathrm{a}}$ XianXi Tang ${ }^{1, \mathrm{~b}}{ }^{*}$ and Jingwei Zhang ${ }^{1, \mathrm{c}}$ \\ ${ }^{1}$ School of civil engineering, Lanzhou University of Technology, Lanzhou, China \\ a 804598375 @qq.com, b695913483@qq.com, ${ }^{c} 760943815$ @qq.com
}

Keywords: Consolidation logarithmic fitting method, logarithmic matching method, settlement evaluation, tunnel engineering

\begin{abstract}
The settlement evaluation of the underline engineering is a vital link to satisfy the high speed, high comfort and high safety of the railway construction. On the basis of Technical Specifications for Observation and Evaluation of Settlement Deformation in Railway Engineering, consolidation logarithmic fitting method is applied to evaluate settlement deformation of a project in Northwest China. Through the analysis of the measured data and the evaluation results, the scientific and practical application of the evaluation method in tunnel engineering is discussed.
\end{abstract}

\section{Introduction}

Settlement evaluation of underline engineering is an important part of Railway Engineering construction. The passenger dedicated line has a very high demand for the settlement evaluation of the project. With the development of railway construction in our country, ballastless track is widely used. The ballastless track railway is especially strict for passenger dedicated line in the deformation of subgrade, bridge and culvert as well as tunnel. The post construction settlement of the underline project needs to be predicted and evaluated, and the track can be laid when those conditions are satisfied. It is very important to choose the appropriate method to evaluate the deformation of structure. Based on the measured data, this paper mainly discusses the application of consolidation logarithmic fitting method in settlement evaluation.

\section{Assessment Method and Applicable Standards}

\subsection{A Brief Introduction to Consolidation Logarithmic Fitting Method}

The consolidation logarithmic fitting method is also called the three point method. It can be applied to any stage of the project. The theoretical solution of the degree of consolidation is expressed as:

$$
U=1-\alpha \cdot \mathrm{e}^{-\beta \mathrm{t}}
$$

According to the definition of consolidation degree:

$$
U_{\mathrm{t}}=\frac{S_{\mathrm{t}}-S_{\mathrm{d}}}{S_{\infty}-S_{\mathrm{d}}}
$$

Type: $S_{d}$-Instantaneous settlement

$$
S_{\infty} \_ \text {Final settlement }
$$

Simultaneous equations of the above two equations:

$S_{\mathrm{t}}=S_{\mathrm{d}} \alpha e^{-\beta t}+S_{\infty}\left(1-\alpha e^{-\beta t}\right)$

In order to obtain the settlement at time $t$ equals $t$, three points on the time curve of initial 
settlement, $\left(t_{1}, S_{1}\right),\left(t_{2}, S_{2}\right)$ and $\left(t_{3}, S_{3}\right)$, can be substituted into the upper equation respectively, and Adding the equation of $t_{3}-t_{2}=t_{2}-t_{1}$, instantaneous settlement $S_{d}$ and final settlement $S_{\infty}$ with parameters can be solved by simultaneous equations. In that expression, $\alpha$ Variable can be calculated by theoretical value or measured data (theoretical value is used in this paper, $\alpha=\frac{8}{\pi^{2}}$ ). A settlement at any time can be obtained by taking the obtained $\beta, S_{\infty}$, $S_{d}$ into the equation (3) respectively.

The concrete solution process is as follows:

$$
\begin{aligned}
& S_{1}=S_{\infty}\left(1-\alpha \mathrm{e}^{-\beta \mathrm{t}_{1}}\right)+S_{\mathrm{d}} \alpha \mathrm{e}^{-\beta \mathrm{t}_{1}} \\
& S_{2}=S_{\infty}\left(1-\alpha \mathrm{e}^{-\beta \mathrm{t}_{2}}\right)+S_{\mathrm{d}} \alpha e^{-\beta t_{2}} \\
& S_{3}=S_{\infty}\left(1-\alpha e^{-\beta t_{3}}\right)+S_{d} \alpha e^{-\beta t_{3}}
\end{aligned}
$$

So the solutions are:

$$
\begin{aligned}
& e^{\beta\left(t_{1}-t_{2}\right)}=\frac{S_{2}-S_{1}}{S_{3}-S_{2}} \\
& \beta=\frac{1}{t_{1}-t_{2}} \ln \frac{S_{2}-S_{1}}{S_{3}-S_{2}} \\
& S_{\infty}=\frac{S_{3}\left(S_{2}-S_{1}\right)-S_{2}\left(S_{3}-S_{2}\right)}{\left(S_{2}-S_{1}\right)-\left(S_{3}-S_{2}\right)} \\
& S_{d}=\frac{S_{t}-S_{\infty}\left(1-\alpha e^{-\beta t}\right)}{\alpha e^{-\beta t}}
\end{aligned}
$$

\subsection{Applicable Standards}

According to the Technical Regulations for Observation and Evaluation of Settlement and Deformation in Railway Engineering, when the correlation coefficient of curvilinear regression method is no less than 0.92, the trend of settlement deformation determined is reliable. Therefore, a correlation coefficient of 0.92 is used as a measure of the applicability of the evaluation method in this paper. The correlation coefficients in this paper are also defined by mathematical statistics:

$$
R=\frac{\operatorname{Cov}(\bar{X}, \bar{Y})}{\sqrt{D(\bar{X})} \sqrt{D(\bar{Y})}}
$$

Type: $\bar{X}, \bar{Y} \longrightarrow$ Sample mean

$$
\begin{aligned}
& \operatorname{Cov}(\bar{X}, \bar{Y})-\text { Covariance } \\
& D(\bar{X}) 、 D(\bar{Y}) \text { _ Variance }
\end{aligned}
$$

First, the expression of the settlement curve is obtained by using the measured data, then the fitting data of the corresponding time point is calculated, and then the correlation coefficient between the predicted and actual values of the two sets of data is calculated by using the function CORREL in EXCEL.

\section{Case Studies}

\subsection{Project Overview}

The tunnel is located in the northwest province of our country, the beginning and ending mileage 
is DK194+980 to DK215+080. This line gets into Dang Jinshan station out of the tunnel. Within $470 \mathrm{~m}$ from the entrance, it is a double track tunnel and the rest is single. The tunnel is uphill on one side, and the slope of the hole is $6 \%, 12.3 \%$ and $7 \%$ respectively. Slope of construction section in $3 \#$ incline opening is $12.3 \%$. The strata passed through by tunnel mainly include Quaternary, upper Pleistocene, pluvial fine breccia soil, Lower Silurian mica quartz schist and so on. The foundation of this tunnel is ballastless track bed.

\subsection{Assessment Criteria}

(1)The observation data obtained no less than 3 months after the completion of the main project is reasonable in the initial assessment of tunnel. Moreover, it is necessary that no less than 3-months intervals between the initial assessment and the second assessment are reserved.

(2) When the geological condition is good, the settlement tends to be stable, as well as the total amount of design and measured settlement is not greater than $5 \mathrm{~mm}$, the conclusion can be obtained that the it meets the laying condition of Ballastless track.

(3) The final settlement prediction time shall satisfy the following conditions:

$$
\frac{S(t)}{S(t=\infty)} \geq 75 \%
$$

Type: $S(t) \longrightarrow$ Predicted settlement

$$
S(t=\infty)-\text { Final settlement }
$$

Note: The completion of invert construction is taken as the starting point for both calculations of settlement and time.

(4) The settlement value of the predicted tunnel foundation shall not be greater than $15 \mathrm{~mm}$ after completion of the settlement.

\subsection{Settlement Deformation and Predictive Analysis}

Taking the observation data from an actual tunnel section as an example in a passenger dedicated line, this paper studied the application of consolidation logarithmic fitting method. First, the few abnormal data is discarded artificially. Then, the results of settlement analysis are obtained by solving above equations. Finally, the correlation coefficient is calculated, and the applicability of consolidation logarithmic fitting method will be analyzed.

\begin{tabular}{|c|c|c|c|c|}
\hline \multirow[b]{2}{*}{$\begin{array}{l}\text { Section } \\
\text { number }\end{array}$} & \multirow{2}{*}{$\begin{array}{l}\text { Predicted } \\
\text { settlement } \\
\text { value } / \mathrm{mm}\end{array}$} & \multicolumn{3}{|c|}{ Consolidation Logarithmic Fitting Method } \\
\hline & & $\begin{array}{c}\text { Prediction of } \\
\text { final settlement } \\
\text { mm }\end{array}$ & $\begin{array}{l}\text { Correlation } \\
\text { coefficient }\end{array}$ & $S_{t} / S_{\infty}$ \\
\hline 0209989S2 & 1.84 & 2.25 & 0.996 & 0.82 \\
\hline 0210506S1 & 0.93 & 0.97 & 0.998 & 0.96 \\
\hline 0210954S2 & 1.22 & 1.33 & 0.984 & 0.92 \\
\hline 0212601S2 & 1.19 & 1.35 & 0.996 & 0.88 \\
\hline 0214220S2 & 1.49 & 1.93 & 0.996 & 0.77 \\
\hline 0214980S1 & 1.38 & 1.73 & 0.987 & 0.80 \\
\hline 0215040S2 & 1.15 & 1.30 & 0.983 & 0.88 \\
\hline
\end{tabular}

Table 1 Settlement analysis of calculation results

As can be seen from Table 1, the correlation coefficients of each section calculated by the consolidation logarithmic fitting method are all greater than 0.92 , which meets relevant requirements. In addition, the values of $S_{t} / S_{\infty}$ in Table 1 are all greater than $75 \%$, which also meets 
the requirements and the applicability of consolidation logarithmic fitting method in tunnel engineering is verified.

\section{Conclusion}

1.Through the prediction analysis in several sets of early data, the correlation coefficient of curvilinear regression is not less than 0.92 , and the values of $S_{t} / S_{\infty}$ are all greater than $75 \%$, which proved that consolidation logarithmic fitting method is applicable to the settlement evaluation and prediction in the early stage of engineering.

2. Three points at the same time period on the settlement-time curve need to be selected in consolidation logarithmic fitting method. The measured initial settlement data must be much enough to solve the coefficients in the Formula (3), which is also a drawback of consolidation logarithmic fitting method.

3. There are many methods for settlement assessment and prediction in a project. The key to finding a suitable assessment method is that the data to be taken must have sufficient observation accuracy, so that the evaluation results can be accurate and reliable.

\section{Reference}

[1] Ministry of railways. Technical Specifications for Observation and Evaluation of Settlement and Deformation in Railway Engineering [S] Beijing, China Railway Publishing House, 2016 (No. 186) 